\title{
The clinical utility of next-generation sequencing in the neonatal intensive care unit
}

\author{
Sarah C. Bowdin MD MSc
}

See also page E254 and www.cmaj.ca/lookup/doi/10.1503/cmaj.150823

Competing interests:

None declared.

This article was solicited and has not been peer reviewed.

Correspondence to: Sarah Bowdin, sarah. bowdin@sickkids.ca

CMAJ 2016. DOI: 10.1503 /cmaj.160490
$\mathrm{F}$ or infants with suspected genetic or metabolic conditions who are admitted to the neonatal intensive care unit, an accurate and timely diagnosis allows management decisions to be based upon the likely disease trajectory and can also allow patients to benefit from the early provision of disease-altering therapies. ${ }^{1}$ The current diagnostic process for most of these infants is slow, invasive and costly, with many succumbing to their condition or being discharged after a long stay but without a unifying diagnosis.

The authors of a linked research paper describe their experience with a pilot project that used a next-generation sequencing panel of 4813 "clinically relevant" genes to enable a specific genetic diagnosis in 8 of 20 neonates in whom a genetic or metabolic condition was suspected. ${ }^{2}$ The authors' aim was not to provide a rapid genetic result; however, the medical or surgical management of two patients was substantially altered because of an accurate genetic diagnosis. The complex presenting features of neonates in this cohort ranged from acute renal failure to multiple congenital anomalies with early-onset seizures, exemplifying the nonspecific phenotypes that neonatologists and their consulting specialist colleagues regularly face.

In all 20 babies studied, multiple additional clinical investigations were ordered concomitantly, and only two of the eight diagnoses made using the next-generation sequencing panel for "clinically relevant disease" were also made as a result of the clinical tests ordered. This finding

\section{KEY POINTS}

- Next-generation sequencing technology is enabling more accurate genetic diagnoses to be made in acute medical settings.

- Accurate interpretation of next-generation sequencing results requires a close partnership among clinicians, molecular geneticists and bioinformaticians.

- Genetic diagnoses can affect the immediate and longer-term management of critically ill patients in the neonatal intensive care unit.

- An accurate genetic diagnosis in a neonate has important implications for the parents' future reproductive choices and options. bolsters the argument for employing a very broad, yet clinically relevant, gene panel when the patient's phenotype suggests a disease process that is clinically and genetically heterogeneous. The alternative is to employ broader screening tests designed to detect relatively few genetic and metabolic diseases, or to use much narrower disease-specific gene panels with the possibility that the panel will not include the gene responsible for the baby's condition.

For one individual in the linked study, the specific diagnosis of a mutation in the Wilms tumour 1 gene (WT1) allowed the choice of a potentially life-saving intervention (bilateral nephrectomy). ${ }^{2}$ For other families, accurate diagnosis may allow initiation of a discussion regarding comfort care rather than full intervention or further invasive procedures. These outcomes are equally important, since they allow families to make informed decisions on the basis of more accurate prognostic information. Having an accurate diagnosis at the earliest point possible removes many of the uncertainties about the appropriateness of a treatment course or direction.

It is possible to use next-generation sequencing technologies to make a rapid genetic diagnosis. In a seminal study from the neonatal intensive care unit at the Children's Mercy Hospital in Kansas City, Missouri, the authors showed that accurate molecular results could be obtained in 50 hours. $^{3}$ Such a short turnaround time requires a dedicated team of clinicians, bioinformaticians and molecular geneticists working together and, importantly, involves close liaison among all members of the team.

No longer should a clinician expect to send a DNA sample to the molecular genetics laboratory with minimal clinical information, since accurate interpretation of the thousands of genetic variants in any large-scale sequencing experiment is an iterative process. The clinicians caring for the patient are uniquely placed to integrate their knowledge of the patient's phenotype with the genetic variants or mutations detected, with the ultimate aim of 
matching the phenotype to the genotype and helping to prevent false-positive or falsenegative results. ${ }^{4}$ In some instances, a definitive molecular diagnosis can be a relatively straightforward outcome of genomic sequencing, since the uniqueness of the patient's phenotype and the pathogenicity of the mutations will leave the molecular geneticist and the clinician in no doubt. However, some cases are solved only with further phenotyping of the patient according to a suggested or "differential" molecular diagnosis, a process sometimes termed "reverse phenotyping." In these instances, the importance of a direct communication channel between members of the interpretation team cannot be overstated. This principle already holds true for all clinicians who order next-generation sequencing genetic test panels, but will become more important as the number of genes tested (and hence the number of variants detected) increases.

For all genetic diagnoses, the clinical utility goes beyond individualizing acute management or designing evidence-based surveillance programs. Enabling the family to understand why their baby is ill can help to assuage the almost universal guilt felt by parents that they did something wrong to cause their baby's illness. It can also indicate whether other family members may be at risk of the same disease and provide an accurate recurrence risk for future pregnancies.

The importance of providing the family with a diagnosis even after a baby has died should not be underestimated, because some couples may decide not to have more children unless the same disease can be avoided. The options now available to couples wishing to pursue a further pregnancy include preimplantation genetic diagnosis and standard prenatal diagnostic techniques, such as chorionic villus biopsy and amniocentesis. None of these tests can be offered for future pregnancies if the molecular basis of the disease in the affected baby is not known; hence, accurate genetic diagnosis of the neonate also has far-reaching implications for the parents' future reproductive options and choices.

Next-generation sequencing of increasingly large gene panels is set to become a standard of care in many adult and pediatric diagnostic settings, especially those where making the diagnosis is highly time sensitive. Across Canada, local expertise in genomic sequencing and interpretation will be built around established molecular genetics laboratories and clinical genetics services; however, building new partnerships with ordering clinicians (such as neonatologists and intensive care physicians) will be integral to unlocking the diagnostic power of next-generation sequencing.

\section{References}

1. Petrikin JE, Willig LK, Smith LD, et al. Rapid whole genome sequencing and precision neonatology. Semin Perinatol 2015;39:623-31.

2. Daoud H, Luco SM, Li R, et al. Next-generation sequencing for diagnosis of rare diseases in the neonatal intensive care unit. CMAJ 2016;188:E254-60.

3. Saunders CJ, Miller NA, Soden SE, et al. Rapid whole-genome sequencing for genetic disease diagnosis in neonatal intensive care units. Sci Transl Med 2012;4:154ra135.

4. Bowdin SC, Hayeems RZ, Monfared N, et al. The SickKids Genome Clinic: developing and evaluating a pediatric model for individualized genomic medicine. Clin Genet 2016;89:10-9.

Affiliation: Division of Clinical and Metabolic Genetics, Hospital for Sick Children, Toronto, Ont.

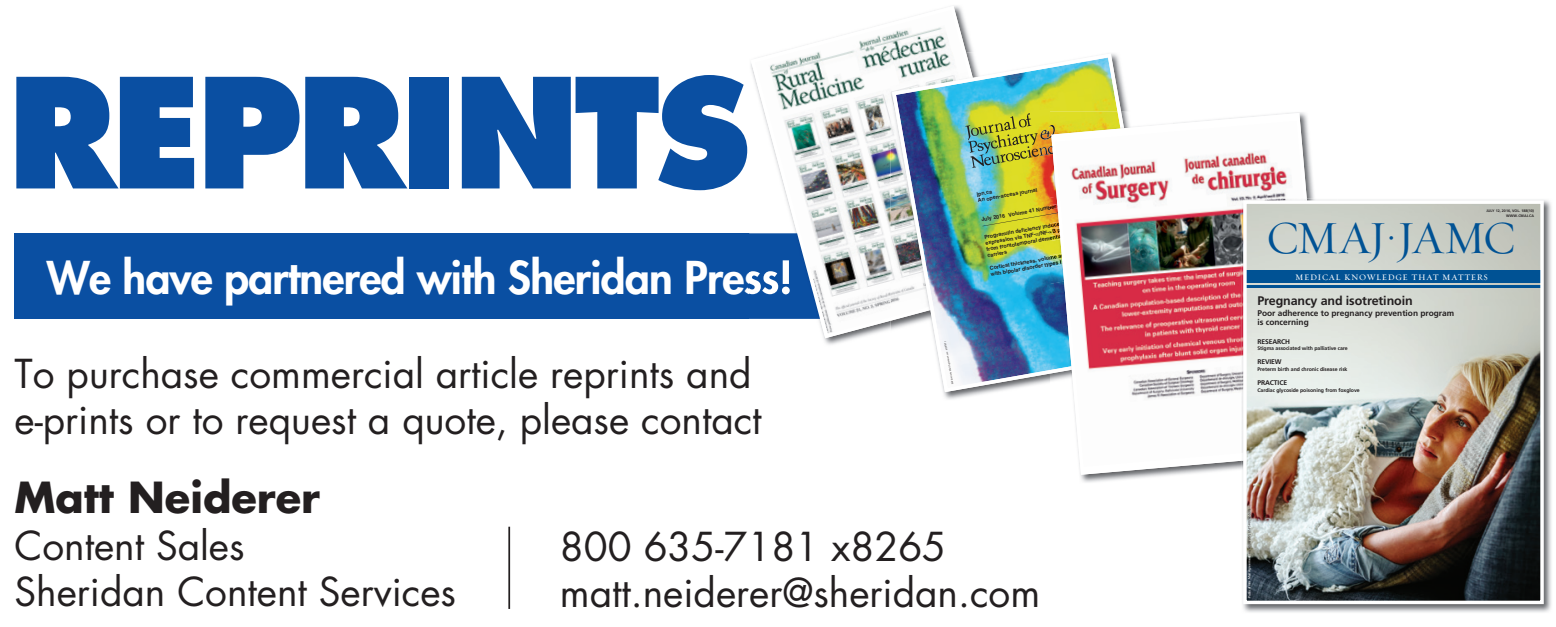

